\title{
Improved Hole-Transporting Properties in Conjugated Polymers Mixed with Polystyrene as an Insulating Polymer
}

\author{
Yuya Horiuchi, Koshiro Midori, Hyung Do Kim*, and Hideo Ohkita* \\ Department of Polymer Chemistry, Graduate School of Engineering, Kyoto University, \\ Katsura, Nishikyo, Kyoto 615-8510, Japan. \\ *hyungdokim@photo.polym.kyoto-u.ac.jp \\ *ohkita@photo.polym.kyoto-u.ac.jp
}

\begin{abstract}
Herein, we study hole transport properties in various conjugated conducting polymers blended with polystyrene (PS) as an insulating polymer. By analyzing macroscopic current density-voltage characteristics, we found that the mobility is improved for the conjugated conducting polymers diluted in the PS matrix that exhibit a redshift in absorption spectra. This is probably ascribed to more ordered polymer chains caused by the addition of PS. We believe that such a dilution of polymer chains would be versatile strategies to enhancing charge transport properties, which will offer a breakthrough towards highly efficient wearable optoelectronic devices based on the conjugated polymers.
\end{abstract}

\section{Keywords: Charge transport properties, Conjugated polymers, Insulating polymers,} Blend films.

\section{Introduction}

Flexible and wearable optoelectronic devices, such as light-emitting diodes, photovoltaic devices, and photodetector devices, have recently attracted much attention owing to their potential applications in modern society. ${ }^{[1-3]}$ Among all materials employed in these devices, conjugated conducting polymers are used as active layers in common and play a central role in transporting of charge carriers. $^{[4-6]}$ With the aim of their commercialization, one of most important issues in this community is, therefore, to achieve highly efficient charge transport in organic materials comparable to that in inorganic materials. For organic solar cells and organic photodetectors, in particular, it is more important to comprehend charge transport properties in blend films rather than in neat films since they are typically composed of an electron donor material (D) and an electron acceptor material (A).

Such blend films, however, are typically accompanied by changes in the degree of structure order and/or the packing orientation of polymer chains. This has a positive or negative impact on their charge transport properties depending on miscibility of polymers employed. For example, in the case of the polymer solar cells, it is found that a fill factor (FF), which is a criterion of charge transport in devices, is much lower in the ternary blend polymer solar cells based on $\mathrm{D} / \mathrm{D} / \mathrm{A}$ or $\mathrm{D} / \mathrm{A} / \mathrm{A}$ than that in D/A binary blend counterparts. ${ }^{[7,8]}$ This degradation results from a decrease in carrier mobility $\mu$ due to the formation of more complicated transport paths incurred by the addition of third components. On the other hand, an opposite trend for charge transport has been recently reported by several groups including ours. ${ }^{[9-14]}$ To be more specific, FF for the ternary blend solar cells was improved by the introduction of the third component as a donor or acceptor material in comparison with that for the binary blend devices. This is ascribed to more balanced $\mu$ between electrons and holes and/or enhanced $\mu$ as a result of more ordered structure of polymer chains in the ternary blend films. For such blend films, however, there would be some possible variations in the charge carrier density $n$ caused by charge transfer (CT) complex at the D/A interfaces. ${ }^{[14]}$ This makes it difficult for us to 
disclose the origin of an improvement in charge transport properties in the blend films. On the other hand, we can focus on charge transport properties in the conjugated polymers blended with an insulating polymer without consideration of variations in $n$. Very recently, several groups including ours have reported that $\mu$ in the conjugated polymers diluted with insulating polymer matrix is higher compared to the conjugated polymer neat films. ${ }^{[15-20]}$ This is attributed to a difference in molecular interactions and/or aggregation states between polymer chains, which are closely related to trap sites unfavorable for charge transport properties. However, little is known about universal criteria for effects of improvement in charge transport properties of the conjugated polymers induced by PS addition.

Herein, we have studied charge transport properties in a series of conjugated polymers blended with PS. More specifically, we employed poly[4,8bis(5-(2-ethylhexyl)thiophen-2-yl)benzo[1,2-b:4,5$\left.b^{\prime}\right]$ dithiophene-co-3-fluorothieno[3,4-b] thiophene2-carboxylate] (PTB7-Th), poly[2-methoxy-5-(3', $7^{\prime}$ -dimethyloctyloxy)-1,4-phenylenevinylene] (MDMO-PPV), poly[2,5-bis(3', 7' dimethyloctyloxy)-1,4-phenylenevinylene (DMOPPV), and regiorandom poly(3-hexylthiophene) (RRa-P3HT) as conjugated polymers, and PS as an insulating polymer. Figure 1 shows chemical structures of these materials. For these blend films,

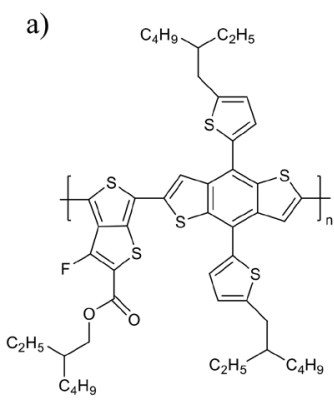

c)

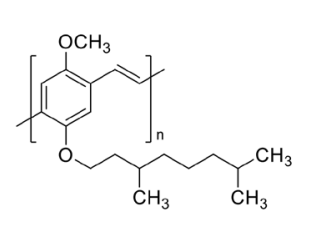

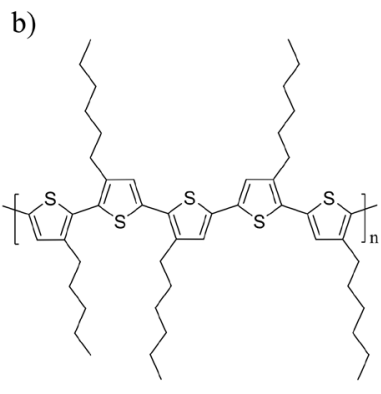

d)

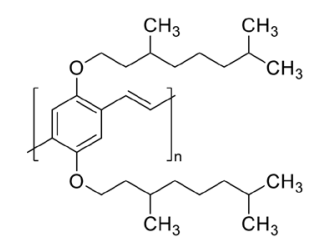

Fig. 1. Chemical structures of materials employed in this study: a) PTB7-Th, b) RRa-P3HT, c) MDMOPPV, and d) DMO-PPV. $\mu$ was evaluated from macroscopic current densityvoltage $(J-V)$ characteristics. As a result, we found that $\mu$ is higher in the blend films except for RRaP3HT/PS blends rather than in the neat films. The origin of an increase in $\mu$ was discussed by measuring absorption spectra and conductive atomic force microscopy (C-AFM) images of these blend films.

\section{Experimental}

\subsection{Materials}

Four kinds of the conjugated polymers employed were PTB7-Th (1-Materials Inc., weight average molecular weight, $\left.M_{\mathrm{w}}=180,000\right)$, MDMO-PPV (Sigma-Aldrich), DMO-PPV (Sigma-Aldrich), and RRa-P3HT (Sigma-Aldrich). Polystyrenes with different molecular weights were purchased from Shodex $\left(M_{\mathrm{w}}=3,250,22,500\right.$, and 1,310,000). As a buffer layer, poly(3,4-ethylenedioxythiophene): poly(4-styrenesulfonate) (PEDOT:PSS, H.C. Starck Clevios PH500) was employed. All these materials employed were used without further purification.

\subsection{Device fabrication}

Hole only devises were fabricated as follows. Indium-tin-oxide (ITO)-coated glass (10 $\Omega$ per square) substrates were cleaned by ultrasonication with toluene, acetone, and then ethanol for $15 \mathrm{~min}$ each. The substrates were dried under nitrogen gas flow, and exposed to ozone under ultraviolet illumination for $30 \mathrm{~min}$ (Nippon Laser \& Electronics, NL-UV253S). The solution of PEDOT:PSS was filtered with a PTFE syringe filter (pore size: 0.45 $\mu \mathrm{m})$ and treated with ultrasonic crusher for $3 \mathrm{~min}$. A layer of PEDOT:PSS was fabricated onto the ITO substrates by spin-coating at a spin rate of $400 \mathrm{rpm}$ for $10 \mathrm{~s}$ and then $3000 \mathrm{rpm}$ for $60 \mathrm{~s}$, and subsequently dried on a hot plate at $140^{\circ} \mathrm{C}$ for $10 \mathrm{~min}$ in air. The mixed solution was prepared by dissolving the conjugated polymer and PS with a different weight ratio of $10: 0,0.75: 0.25,0.4: 0.6$ and $0.2: 0.8$ in chlorobenzene or chloroform. The blend active layers were deposited atop ITO/PEDOT:PSS substrates by spin-coating at $1000 \mathrm{rpm}$ for $60 \mathrm{~s}$ in the nitrogen atmosphere. The thickness of blend film was $\sim 100 \mathrm{~nm}$. Finally, $\mathrm{Au}(100 \mathrm{~nm})$ or $\mathrm{MoO}_{3} / \mathrm{Al}$ $(10 \mathrm{~nm} / 100 \mathrm{~nm})$ electrode was thermally evaporated on top of the active layer. Consequently, the device layered structure was as follows: ITO/PEDOT:PSS/active layer/Electrode.

\subsection{Measurement}

$J-V$ characteristics were measured with a direct- 
current (DC) voltage and current source/monitor (Keithley, 2611B) in the dark. To evaluate $\mu$, we analyzed space-charge-limited current (SCLC) of the blend films with the Mott-Gurney equation as follows. ${ }^{[21]}$

$$
J=\frac{9}{8} \varepsilon_{\mathrm{r}} \varepsilon_{0} \mu \frac{V^{2}}{L^{3}}
$$

where $\varepsilon_{\mathrm{r}}$ is a relative dielectric constant of materials, $\varepsilon_{0}$ is the vacuum permittivity, and $L$ is a thickness of the blend film. Here, $\varepsilon_{\mathrm{r}}$ is assumed to be 3 , which is a typical value for most organic materials. Absorption spectra were measured with a spectrophotometer (Hitachi, U-4100). The film surface morphology and current image were measured simultaneously with an atomic force microscope (AFM, Shimadzu, SPM-9700) using an Au-coated silicon probe (NANO-SENSORS, PPPCONTAu, tip radius of curvature $<10 \mathrm{~nm}$, spring constant $=0.2 \mathrm{~N} \mathrm{~m}^{-1}$ ) at a constant sample voltage of $+5.0 \mathrm{~V}$. The sample bias was applied to the ITO and the tip was grounded.

\section{Results and discussion}

Figure 2 shows $\mu$ of each donor/PS blend film with different weight ratios, which was evaluated by fitting the $J-V$ characteristics in the SCLC region with a slope of two. For PTB7-Th/PS blends, $\mu$ was comparable to that of PTB7-Th neat films at $25 \mathrm{wt} \%$ $\mathrm{PS}$, the largest at $60 \mathrm{wt} \% \mathrm{PS}$, and still larger than that of the neat films even at only $80 \mathrm{wt} \%$ PS. In other words, all the blend films exhibited $\mu$ comparable to or larger than PTB7-Th neat films. For MDMOPPV/PS blends, $\mu$ increased with increasing PS fraction in the blends. Interestingly, the values of all $\mu$ were larger than that of MDMO-PPV neat films even though the fraction of the conductive polymers was smaller than that of the neat films. For DMOPPV/PS blends, $\mu$ decreased with increasing PS fraction in the blends. However, the decreased $\mu$ was limited and much smaller than that expected from a decrease in the fraction of the conductive polymers. In other words, this finding rather suggests that an effective $\mu$ is improved with increasing PS fraction in the blends considering the volume fraction of the conjugated polymers. For RRa-P3HT/PS blends, on the other hand, $\mu$ significantly decreased by one order of magnitude even at $40 \mathrm{wt} \%$ of conductive polymers where other conjugated polymers exhibit the largest $\mu$. In summary, $\mu$ was improved for PTB7-Th/PS, MDMO-PPV/PS, and DMO-PPV/PS blends while it

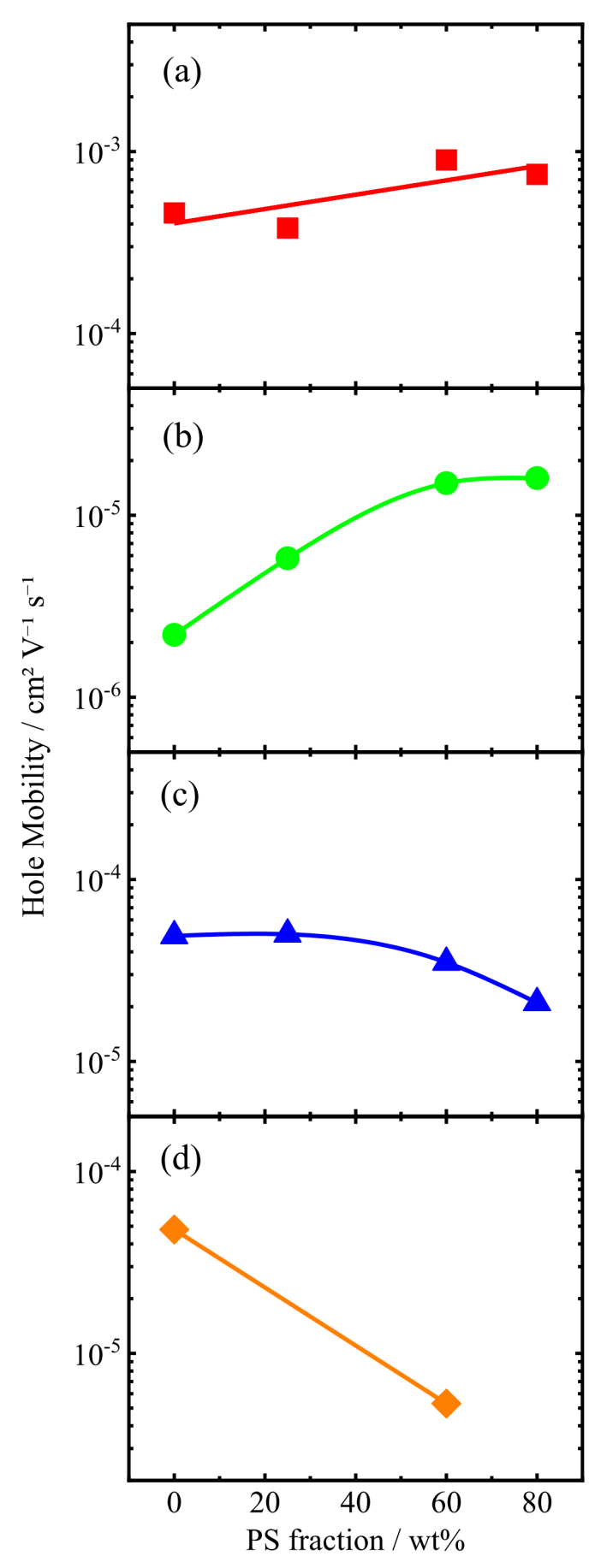

Fig. 2. Hole mobility of each donor/PS blend film with different weight ratio: a) PTB7-Th, b) MDMOPPV, c) DMO-PPV, and d) RRa-P3HT.

decreased for RRa-P3HT/PS blends.

In order to consider how molecular weights of PS matrix impact on charge transport properties, we evaluated $\mu$ for $40 \mathrm{wt} \%$ PTB7-Th in the PS matrix with different PS molecular weights. As a result, we found that $\mu$ decreases from $5.5 \times 10^{-4} \mathrm{~cm}^{2} \mathrm{~V}^{-1}$ $\mathrm{s}^{-1}$ for $M_{\mathrm{w}}=22,000$ to $2.9 \times 10^{-4} \mathrm{~cm}^{2} \mathrm{~V}^{-1} \mathrm{~s}^{-1}$ for $M_{\mathrm{w}}$ 
$=131,000$. Note that the $\mu$ for all the blends are comparable to or larger than that of PTB7-Th neat films. In other words, charge transport is improved for all the blends but is most effectively enhanced for the PS matrix with the smallest molecular weight $\left(M_{\mathrm{w}}=3,250\right)$. Figure 3 shows AFM images of PTB7-Th/PS blends with different molecular weights of PS. For the largest molecular weight of PS matrix, sea and island domains were observed, suggesting large phase separation structures. For the smaller molecular weight of PS matrices, on the
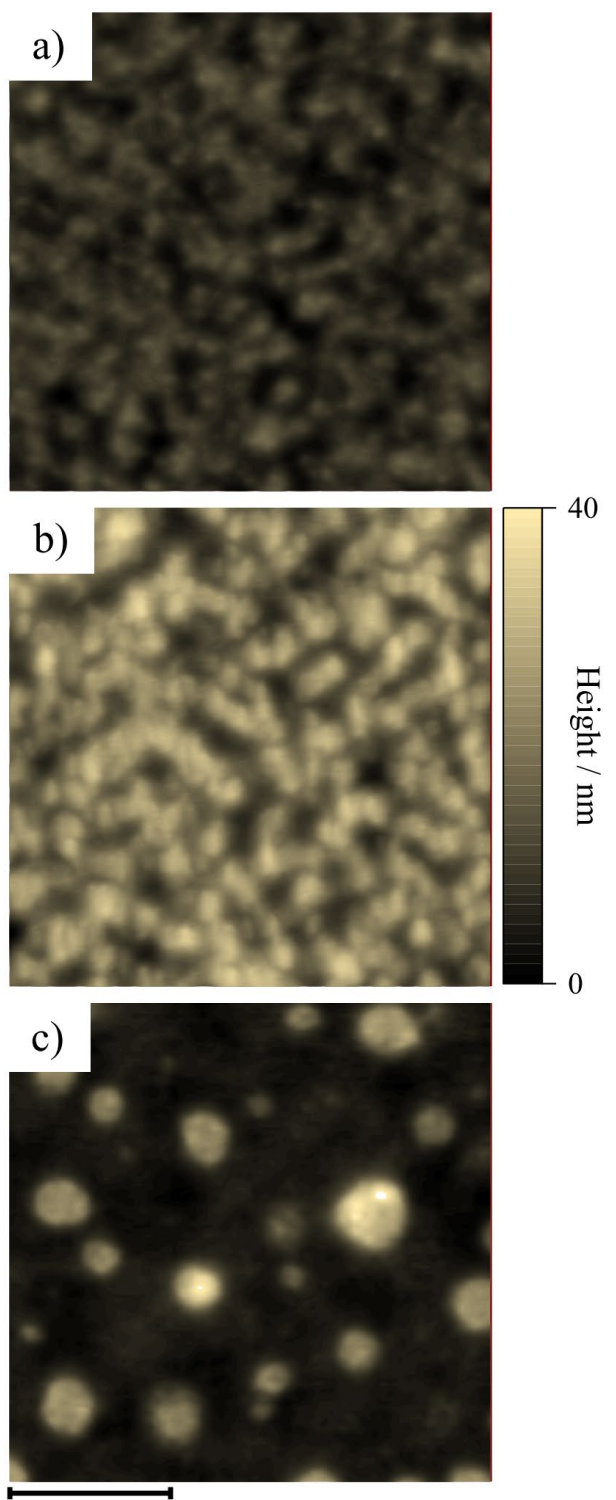

$1 \mu \mathrm{m}$

Fig. 3. AFM images of PTB7-Th/PS blend films with different molecular weights of PS: a) $M_{\mathrm{w}}=$ 3,250, b) $M_{\mathrm{w}}=22,500$, and c) $M_{\mathrm{w}}=1,310,000$. other hand, a well-mixed blend morphology was observed. This finding indicates that it would be beneficial for charge transport in the conjugated polymers blended with PS matrix.

To examine how such a blend morphology impacts on optoelectronic properties of the conjugated polymers mixed in PS matrix, we next measured the absorption spectra of these blend films.

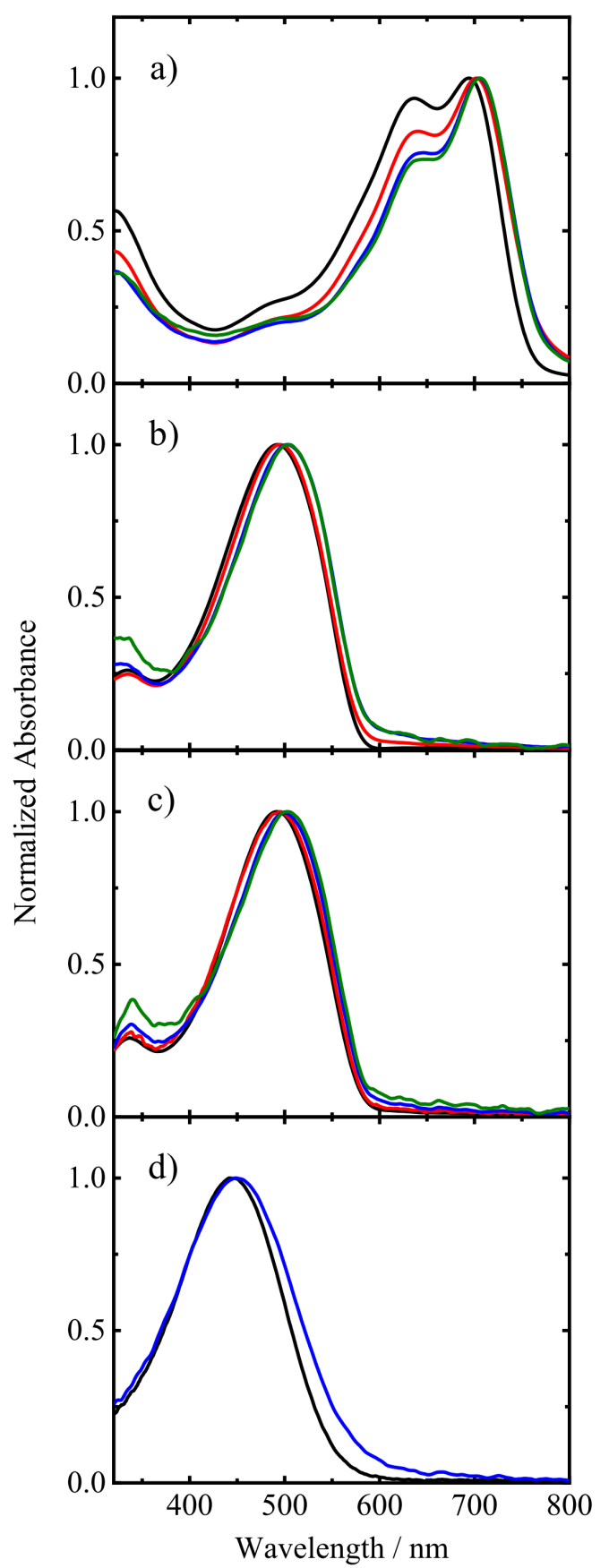

Fig. 4. Absorption spectra of a) PTB7-Th/PS, b) MDMO-PPV/PS, c) DMO-PPV/PS, and d) RRa$\mathrm{P} 3 \mathrm{HT} / \mathrm{PS}$ blend films with different weight ratios: donor : PS $=1: 0$ (black lines), $0.75: 0.25$ (red lines), $0.4: 0.6$ (blue lines), and $0.2: 0.8$ (green lines). 
For PTB7-Th/PS blends, as shown in Figure 4a, an absorption peak was red-shifted and a peak ratio of $0-0$ to $0-1$ vibronic bands increased in comparison with that of PTB7-Th neat films. The same trends were observed even for different molecular weights. This is consistent with our previous report for PTB7Th, suggesting effectively enhanced backbone planarity. ${ }^{[14]}$ For MDMO-PPV/PS and DMOPPV/PS blend films, as shown in Figures $4 \mathrm{~b}$ and $4 c$, absorption peaks were red-shifted in comparison with each neat film, implying improved an effective conjugation length of polymer chains. For RRaP3HT/PS blends, on the other hand, no absorption shift was observed but instead absorption broadening was observed with a more distinct absorption tail as shown in Figure 4d. This finding suggests that inhomogeneities increased in RRa-P3HT/PS blends. In either case, there is a good correlation between charge transport properties and absorption spectra of polymer/PS blend films.

Finally, we studied local current properties on a scale of micrometers by C-AFM measurement. Here, we focus on C-AFM images of MDMOPPV/PS films as an example. For MDMO-PPV neat films, as shown in Figures $5 \mathrm{a}$ and $5 \mathrm{c}$, morphology and current map were homogeneous over the entire films. For MDMO-PPV/PS blend films, on the other hand, sea and island domains were
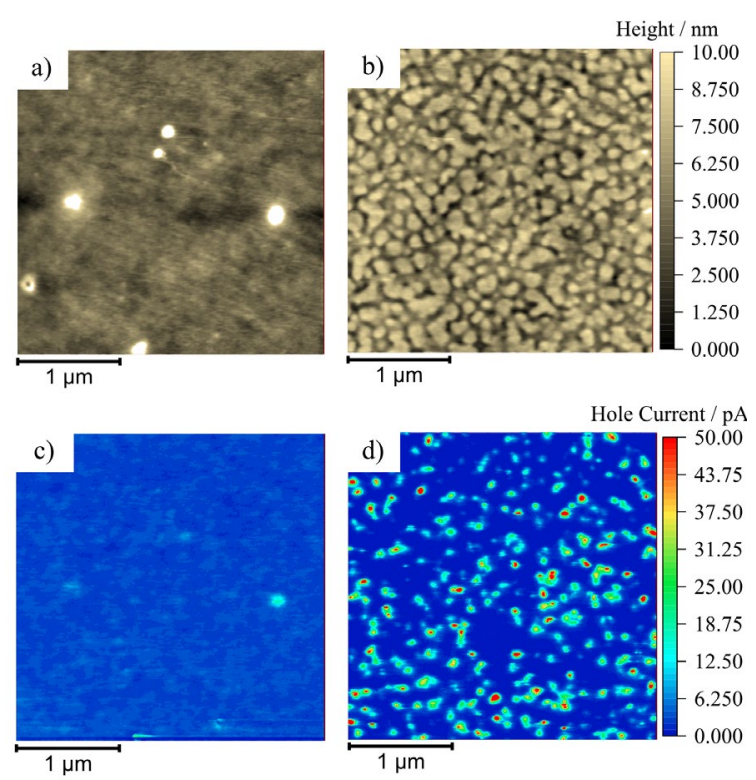

Fig. 5. Surface topographic images (top) and the corresponding hole current images (bottom) of MDMO-PPV/PS blend films with different weight fractions of PS: a) and c) PS $0 \mathrm{wt} \%$ and b) and d) PS $60 \mathrm{wt} \%$. observed in the topography image (Figure 5b). As shown in Figure 5d, several hot spots were observed in the current image: hot spots correspond to islands in the topography image. Thus, island domains can be ascribed to MDMO-PPV rich domains. The pixel ratio between the no current region and the current flow region does not match the blend ratio because of the lateral and/or vertical phase separation for $60 \mathrm{wt} \%$ of PS added blends. Interestingly, currents in hot spots are about 10 times larger than that in sea domains of the blend films or in the neat films. We therefore conclude that an improved charge transport in the polymer/PS blend films is due to the formation of such highly conductive hot spots. Further study is required to reveal polymer structures in hot spots.

\section{Conclusion}

We studied hole transport properties in a series of conjugated polymers blended in PS matrix. As a result, $\mu$ was improved for some polymer/PS blends. This improvement was dependent on molecular weights of PS matrix and more effective in PS matrix with small $M_{\mathrm{w}}$, which gives a well-mixed blend morphology. Interestingly, we found a good correlation between hole transport properties and absorption spectra of the blend films based on a conjugated polymer and PS. More specifically, $\mu$ is improved for the polymer/PS blend films that exhibit red-shifted or enlarged ratio of $0-0$ to $0-1$ bands in absorption spectra: these are characteristic of an improvement in the effective conjugation length and the backbone planarity of the conjugated polymers. We also found such an improved charge transport is due to locally conductive hot spots in the polymer/PS blends on a scale of micrometers.

\section{Acknowledgments}

This study was supported by KAKENHI from Japan Society for the Promotion of Science (JSPS) (Grand Number 19K22217), a project commissioned by the New Energy and Industrial Technology Development Organization (NEDO), the Advanced Low Carbon Technology Research and Development Program (ALCA) of Japan Science and Technology Agency (JST) (Grant Number JPMJAL1404), and Research Grant by Hirose Foundation.

\section{References}

1. H. Ling, S. Liu, Z. Zheng, and F. Yan, Small Methods, 2 (2018) 1800070.

2. S. A. Hashemi, S. Ramakrishna and A. G. Aberle Energy Environ. Sci., 13 (2020) 685. 
3. Y. Lee, H. Zhou, and T. W. Lee, J. Mater. Chem. C, 6 (2018) 3538.

4. C. Zhan, G. Yu, Y. Lu, L. Wang, E. Wujcik, and S. Wei, J. Mater. Chem. C, 5 (2017) 1569.

5. M. Ashizawa, Y. Zheng, H. Tran, Z. and Bao, Prog. Polym. Sci., 100 (2020) 101181.

6. M. Chang, G. T. Lim, B. Park, and E. Reichmanis, Polymers, 9 (2017) 212.

7. H. Kim, M. Shin, and Y. Kim, J. Phys. Chem. C, 113 (2009) 1620.

8. P. Cheng, Y. Lia, and X. Zhan, Energy Environ. Sci., 7 (2014) 2005.

9. L. Nian, Y. Kan, H. Wang, K. Gao, B. Xu, Q. Rong, R. Wang, J. Wang, F. Liu, J. Chen, G. Zhou, T. P. Russell and A. K. -Y. Jen, Energy Environ. Sci., 11 (2018) 3392.

10. L. Lu, W. Chen, T. Xu, and L. Yu, Nat. Commun., 6 (2015) 7327.

11. W. Zhao, S. Li, S. Zhang, X. Liu, and J. Hou, $A d v$. Mater., 29 (2017) 1604059.

12. K. Midori, T. Fukuhara, Y. Tamai, H. D. Kim, and H. Ohkita, ChemPhysChem., 20 (2019) 2683.

13. H. D. Kim, R. Shimizu, and H. Ohkita. Chem. Lett., 47 (2018) 1059.
14. H. D. Kim, R. Iriguchi, T. Fukuhara, H. Benten, and H. Ohkita, Chem. Asian J., 15 (2020) 796.

15. G. Lu, H. Tang, Y. Huan, S. Li, L. Li, Y. Wang, and X. Yang, Adv. Funct. Mater., 20 (2010) 1714.

16. A. D. Scaccabarozzi and N. Stingelin, J. Mater. Chem. A, 2 (2014) 10818.

17. W. H. Lee and Y. D. Park, Polymers, 6 (2014) 1057.

18. B. Tan, H. Pan, H. Li, M. L. Minus. B. M. Budhlall, and M. J. Sobkowicz, J. Phys. Chem. C, 122 (2018) 2918.

19. S. M. Tuladhar, D. Poplavskyy, S. A. Choulis, J. R. Durrant, D. D. C. Bradley, and J. Nelson, $A d v$. Funct. Mater., 15 (2005) 1171.

20. D. Abbaszadeh, A. Kunz, G. A. H. Wetzelaer, J. J. Michels, N. I. Craciun, K. Koynov, I. Lieberwirth, and P. W. M. Blom, Nat. Mater., 15 (2016) 628.

21. N. F. Mott and R. W. Gurney, "Electronic processes in ionic crystals", Oxford University Press, Oxford, 1940. 